\title{
An improved Pulse shape Discrimination (PSD) method to detect directly for a non-baryonic dark
}

\author{
Jong-Kwan Woo ${ }^{1}$ \\ Department of Physics, Jeju National University \\ Jeju National University, Jeju 63243, South Korea \\ E-mail:w00jk@jejunu.ac.kr
}

\section{LIU Dong}

BK21plus Clean Energy Convergence and Integration Center for Human Resources Training and Education

Jeju National University, Jeju 63243, South Korea

E-mail: LIUDONGCN@jejunu.ac.kr

\begin{abstract}
For the several decades, the non-baryonic dark matter has been searched in many ways and by many research teams. Non-baryonic dark matter is known as a weakly interaction and neutral particle. In the direct detection ways, it is not easy to identify a non-baryonic dark matter from other particles, such as gamma ray and neutron, due to its electric neutrality. So, the identification of neutral particle is an essential part for the direct detection method of dark matter in a cosmic ray free laboratory with a detector made of radioactive free materials.

A pulse shape discrimination (PSD) method was introduced more than fifty years ago. And it has been used by various elementary particle experiments. We need a more precise PSD method to detect directly dark matter interacting rarely.

We can see the primary and the secondary signals in the scintillation produced by an elastic scattering between an incident particle and a target atom. The primary signal is composed of the scintillations from an excitation process of target nuclear and an ionization process of target atom while the secondary signal is composed of scintillation from ionization process, only. The ratio of scintillations from excitation and ionization processes gives the clue to identify the neutral particles.

Here, we introduce an improved way to separate the primary signal into the scintillations induced by excitation and ionization processes through the elastic scattering between a target atom and a non-baryonic dark matter. This separation of primary signal can provide a big hint to identify a nonbaryonic dark matter.
\end{abstract}

XXXIX International Conference on High Energy Physics

4-11 July 2018

Seoul, Korea

\footnotetext{
${ }^{1}$ Speaker

(c) Copyright owned by the author(s) under the terms of the Creative Commons Attribution-NonCommercial-NoDerivatives 4.0 International License (CC BY-NC-ND 4.0).
} 


\section{Introduction}

Weakly Interaction Massive Particle (WIMP), a strong candidate for the Non-Baryonic dark matter, is a neutral particle moving with velocity $270 \mathrm{~km} / \mathrm{s}$ on the Sun's orbit. Due to its neutrality and extremely low event rate, WIMP detection method needs the extremely low back ground laboratory. Even in the low neutron back ground and low cosmic ray laboratory, the high rate gamma ray background from the experimental material and environment should be controlled and rejected quickly. The controlled environmental gamma ray background rate should be lower than WIMP detection rate, $\sim 2$ events $/ \mathrm{kg} /$ year.

\section{Mechanism of Pulse Shape Discrimination (PSD) Method}

When an incident particle scatters elastically with target nucleus, scintillations are emitted via an excitation process and an ionization process. A scintillation from excitation and a part of ionization process produce a photon signal, called primary signal. Another part of scintillation from ionization produced a slow scintillation under the high electric field, called a secondary signal. We are going to concentrated only on the primary signal in this presentation. The contribution ratios of an excitation process and an ionization process to form a primary are different each other for the incident particles, a heavy ionization particle (HIP) or a minimum ionization particles (MIP). We call this discrimination method as PSD. For example, a gamma ray belongs to MIP and a neutron belongs to HIP. And, we estimate that a WIMP belongs to HIP. So, we can easily discriminate a gamma ray back ground from the WIMP signal in the WIMP search experiment. Figure 1 shows the typical pulse shapes produced by incidents particles, MIP and HIP.

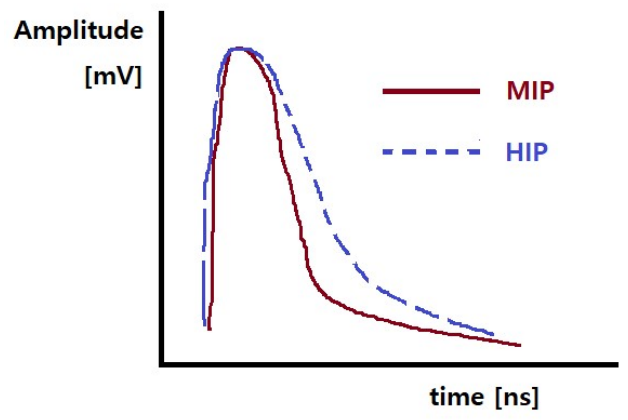

Fig. 1 Typical pulse shapes produced by incidents particles, MIP and HIP.

\section{Analysis and Summary}

The pulse shape, shown in Fig.1, is composed of a scintillation from excitation process and a scintillation from ionization. We assume a scintillation from ionization process is provided slower than a scintillation from excitation process. In other words, a pulse shown in Fig. 1 is a summation of a fast scintillation from excitation and a slow scintillation from ionization. We are trying to find out the actual contributions from two scintillations. For the analysis the contributions from two scintillations, we have to know the shape of signal from the two processes and a time interval between a fast signal and slow signal. The accurate way to discriminate the scintillations induced by gamma ray and HIP will give the clue to find out WIMP dark matter. 